\title{
High-permittivity polymer nanocomposites: Influence of interface on dielectric properties
}

\author{
Zhi-Min Dang* ${ }^{*}$, Jin-Kai Yuan ${ }^{\dagger}$, Jun-Wei Zha*, Peng-Hao Hu*, \\ Dong-Rui Wang* and Zhong-Yang Cheng* \\ *Laboratory of Dielectric Polymer Materials and Devices \\ School of Chemistry and Biological Engineering \\ University of Science and Technology Beijing \\ Beijing 100083, P. R. China \\ ${ }^{\dagger}$ Laboratoire de Mecanique des Sols, Structures et Maeriaux \\ Ecole Centrale Paris, CNRSUMR8579, PRES UniverSud \\ Grande Voie des Vignes, 92295 Chatenay-Malabry Cedex \\ France \\ ${ }^{*}$ Materials Research and Education Center \\ Auburn University, Auburn \\ AL 36849, USA \\ \$dangzm@ustb.edu.cn
}

Received 16 July 2013; Revised 29 August 2013; Accepted 4 September 2013; Published 10 October 2013

\begin{abstract}
Flexible dielectric composites with high permittivity have been extensively studied due to their potential applications in highdensity energy capacitors. In this review, effects of interface characteristics on the dielectric properties in the polymer-based nanocomposites with high permittivity are analyzed. The polymer-based dielectric composites are classified into two types: dielectric-dielectric (DD, ceramic particle-polymer) composites and conductor-dielectric ( $\mathrm{CD}$, conductive particle-polymer) composites. It is highly desirable for the dielectric-dielectric composites to exhibit high permittivity at low content of ceramic particles, which requires a remarkable interface interaction existing in the composite. For conductor-dielectric composites, a high permittivity can be achieved in composite with a small amount of conductor particle, but associated with a high loss. In this case, the interface between conductor and polymer with a good insulating characteristic is very important. Different methods can be used to modify the surface of ceramic/conductor particles before these particles are dispersed into polymers. The experimental results are summarized on how to design and make the desirable interface, and recent achievements in the development of these nanocomposites are presented. The challenges facing the fundamental understanding on the role of interface in high-permittivity polymer nanocomposites should be paid a more attention.
\end{abstract}

Keywords: Interface; microstructure; dielectric properties; nanocomposites; model.

\section{Introduction}

Dielectrics with high permittivity are widely used in most high-tech fields. ${ }^{1-4}$ With the development of flexible devices/ equipments, high-permittivity dielectrics with excellent flexibility are attracting an intensively attention..$^{5-8}$ To meet this kind of need, a composite with polymer as matrix and inorganic particle as functional fillers is often researched in last three decades. As compared to the conventional dielectric ceramics, the dielectric polymers exhibit a relatively high electric breakdown field and importantly offer processing advantages including low processing-temperature and mechanical flexibility as well as the capability of being molded into various configurations for power electronic devices with reduced volume and weight. ${ }^{9-12}$

The introduction of inorganic particles into polymer matrices to form dielectric composites represents one of the most promising methods to acquire high-permittivity polymer-based composites. The composite approach capitalizes upon the idea that the combination of impressive permittivity of inorganic particles with good dielectric strength of polymers may result in a high-permittivity polymer-based flexible composites. ${ }^{5-12}$ Earlier investigations on dielectric composites concentrated mainly on the microsized particle filled systems, yet they are subsequently found to show some shortages in the dielectric properties due to the surface defects and stress cracking after a long-serving time. ${ }^{13-15}$ The thickness of the microcomposite films is also often limited by the size of microparticles. ${ }^{16,17}$ On the other hand, the continuous trend of structural miniaturization in modern electronics highly demands processing capacitor dielectrics into much thin films.

To avoid the shortcomings mentioned above, the only way is replacing microparticles by nanometer-sized filler to prepare the polymer nanocomposites. The advantages of 
nanocomposites include low filler loading, nanometer sizes, and large internal surface areas. ${ }^{18,19}$ The low nanofiller loadings allow for composite formation without scarifying some of the inherent polymeric properties, e.g., density, flexibility, facile processability. Furthermore, when the interfacial energy barriers from the nanoparticle aggregation driven by van der Waals' forces are overcome, the inter-filler distances in the nanocomposites could be in the range of nanometers and the filler would interact chemically and physically with polymer matrices, resulting in the emerging of intermediate or mesoscopic properties. Such mesoscopic properties at interfaces always bring about unexpected excellent macroscopic properties of nanocomposites. ${ }^{20}$ It has been demonstrated that as the size of filler particles decreases to the nanometer scale, large interfacial areas in the composite between the polymer and nanoparticle would promote the exchange coupling effect through a dipolar interface layer, affording higher polarization levels, dielectric response, and breakdown strength. ${ }^{21-26}$ All these features offer new opportunities for designing entirely different class of dielectric polymer nanocomposites with nanometric interfaces, that could offer higher energy storage density or lower dielectric loss, and improved power dissipation. ${ }^{27}$

For a convenient discussion, herein, the high-permittivity polymer nanocomposites can be divided into two types according to their components: dielectric-dielectric (ceramic particle-polymer) nanocomposites and conductor-dielectric (conductive particle-polymer) nanocomposites, ${ }^{28}$ hereby named as DD nanocomposites and CD nanocomposites, respectively. These composites are in common the 0-3 connection ones and the dielectric constant can be calculated on the basis of different models/formulas. ${ }^{28}$ Particularly, a detailed summarization on these models/formulas can be referred in Ref. 28 in terms of DD and CD composites, respectively. ${ }^{28}$ Except other reasons, the interface is one crucial factor having an effect on dielectric properties of the polymer nanocomposites. Therefore, in this short review, we will focus on analyzing the interface characteristics in two kinds of nanocomposites due to the use of different inorganic (dielectric and conductive) particles. Based on some experimental results and our understanding, the principles are about how to design and acquire the expected interfaces in order to improve the dielectric properties of two kinds of polymer nanocomposites. Of course, issues and challenges regarding the understanding of these nanocomposites and development of high-permittivity polymer nanocomposites are discussed.

\section{Analyzing the Interface Characteristics in Composites}

\subsection{Interface characteristics of inorganic particles/ polymer nanocomposites}

Nanoparticle is usually defined as a microscopic particle with at least one dimension less than $100 \mathrm{~nm}$, which can exhibit characteristics totally different from either atoms or bulk materials. As the particle shrinks in size from micrometric to nanometric scale, especially below $20 \mathrm{~nm}$, the percentage of atoms at the surface of the particle becomes more significant, leading to dramatic change in physical properties, interfacial properties and also agglomeration behavior relative to the bulk materials. $^{29}$

Another main concern along with the size effect in nanoparticles is their special surface characteristics associated with large surface area to volume ratio. The great specific surface area would cause nanoparticles to be more reactive with some other molecules. It has been well established that the dielectric behavior of nanoparticles would be significantly different with that of bulk material and micro-particles, (references for two types of inorganic dielectrics: (1) about $\mathrm{BaTiO}_{3}$, and (2) about CCTO. This is mainly due to the influence of the interaction of interfaces with their surroundings, since these interactions may give rise to a certain degree of polarization and charge separation at the interface. $^{25,27,30}$ The high surface energy of nanoparticles encourages coalescence and largely impairs the uniformity of their nanocomposites, which is not desirable to realize the potential of nanoparticles in dielectric polymer nanocomposites.

In order to fully describe how the interfaces are formed chemically, physically and electrically, Tanaka et al. suggested and proposed a hypothetical multi-layered core model for interfaces in a nanocomposite based on a spherical inorganic particle embedded in a polymer matrix. ${ }^{31}$ The multicore structure involves a bonded layer, a bound layer and a loose layer, as shown in Fig. 1. The bonded layer corresponds to a transition layer with thickness of about $1 \mathrm{~nm}$, which is tightly bonded to both inorganic and organic substances. Such bonding arises from either ionic, covalent or hydrogen bonds, or van der Waals' force. The bound layer is a layer of polymer chains strongly bound and/or interacted to the bonded layer and the surface of the inorganic particle. This layer is much concerned to alter the polymer chain conformation and/or mobility, the degree of chain folding, and other stereographic structures. Its thickness is commonly in the range of $2-9 \mathrm{~nm}$. The morphology and thickness of this layer are strongly dependent on the interfacial interaction strength in the bonded layer. It is postulated that tight binding such as ionic or covalent bonding will create a thick bound layer with highly ordered structure around the bonded layer. The loose layer is a region loosely coupling to the bound layer; yet in that layer chain conformation and mobility, and even free volume or crystallinity differs from the polymer matrix. It has a larger thickness of several tens of nm than the inner layers. Reminding that the interfiller spacing is in the same order of magnitude with the particle size in a nanocomposite loaded with several wt.\% of nanofillers, the interface of a nanoparticle is then prone to be overlapped with that of its nearest nanoparticle. In this case, the neighboring core layers are mutually superimposed, giving rise to a zone 


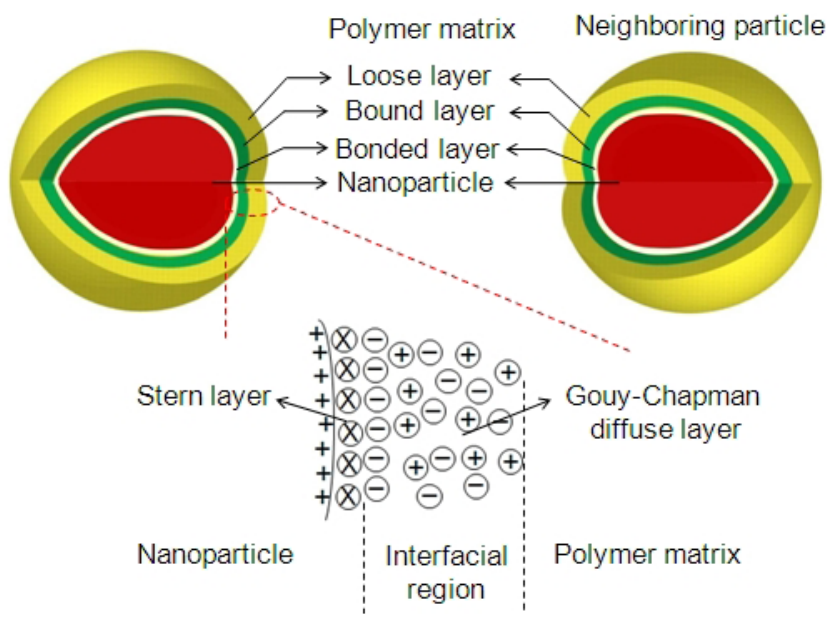

Fig. 1. Multi-core model for interfaces between inorganic nanoparticles and polymer matrix in a polymer nanocomposite. ${ }^{31}$

quite different from the filler and polymer matrix. It is anticipated that this interfacial overlapping phenomenon causes some collaborative effect among neighboring particles (farfield effect) so as to intensify the effect of interfaces on the macroscopic properties of the nanocomposites.

In fact, the morphology of interfacial layers is not only affected chemically by the surface characteristics of nanoparticles, but also electrically by Gouy-Chapman diffusion layer formed, when charges are injected. ${ }^{31}$ As shown in Fig. 1, there is an interfacial region (charge distribution) between the nanoparticle and the polymer matrix in this model. The nanoparticle becomes charged possibly as a consequence of the equalization of Fermi level or chemical potential that causes ionization of surface groups as well as adsorption of ions from the polymer matrix. The segments of polymer chains localized at the interfacial region then responds by establishing a screening diffuse double layer with excess counter charges. ${ }^{25}$ Two mechanisms have been evoked for this response. The first one is concerned with the polymer polarization involving both electronic polarization and the orientation of permanent dipoles. The second one is associated with a diffuse electrical double layer consisting of positive and negative ions, if polymer is solid electrolyte and contains mobile charges. In this layer, charge density decays exponentially with distance according to Born approximation. Debye shielding length of the Gouy-Chapman diffusion layer could be calculated as several tens to $100 \mathrm{~nm}$, indicating the nanoparticles may also interact electrically with the nearest neighbors each other to result in possible collaborative effect. Therefore, it is plausible that the energy storage capacity of the dielectric polymer nanocomposites largely drives from the interfacial polarization that is mainly associated with the bonded layer. Meanwhile, the bound and loose layers also influence the energy density either by the local morphology change as a result of adhesion with bonded layer or through the far-field effect between the neighboring particles.

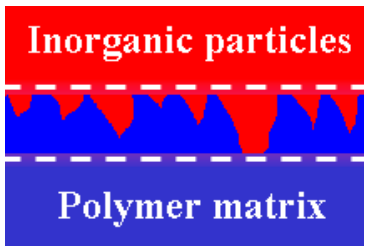

(a)

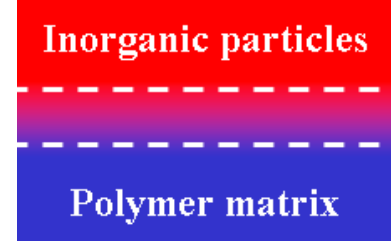

(b)
Fig. 2. (a) A real interface and (b) an ideal interface in the inorganic particles/polymer nanocomposites.

On the basis of presentation above, the interfaces of dielectric polymer nanocomposites may be extremely complicated. It is not a simple bonding directly between nanoparticles and polymer as shown in Fig. 2(a) and will induce an important effect on the dielectric properties of the nanocomposites. In fact, some crucial factors including with and without interface, strong and weak of interfacial action and physical and chemical characteristics will have an undoubted effect on the dielectric properties. Therefore, an interface near to ideal characteristics should be paid more attention as shown in Fig. 2(b), in which the physical and chemical characteristics should be changed gradually and slowly. However, the interface characteristics should be different in the DD nanocomposites and the CD nanocomposites, due that the principle for improving the dielectric constant is different in these kinds of composites. One is based on the dielectric-dielectric coupling effect and another is percolation effect. $^{32-35}$

\subsection{Interface characteristics in the $D D$ and $C D$ nanocomposites}

Due to the different mechanisms for improving the dielectric constant, different interface characteristics in the DD nanocomposites and $\mathrm{CD}$ nanocomposites should be created. In fact, for the DD nanocomposites, the increase in dielectric permittivity is almost the same when the dielectric inorganic nanoparticles with remarkably different permittivity are employed at the same loading content. This is because the permittivity of polymers is commonly below 5 . However, the permittivity of inorganic fillers is generally above $10^{3}$ as much as $10^{4}$. In this case, the permittivity gradient across the interface between filler particles and polymer matrix is very large as shown in Fig. 3(a). This results in the fact that there is not much difference in the permittivity of the DD nanocomposites using different dielectric fillers. Therefore, the interface with large permittivity gradient as barrier interphase layer should have an important effect on improving the dielectric properties of the DD nanocomposites. In addition, the content of inorganic particles should be as low as possible so that the DD nanocomposites can be endowed with an excellent flexibility. In this case, an effective dielectricdielectric coupling at interface is extremely crucial in order to acquire a high permittivity at low loading of inorganic 
particles. Therefore, an ideal interface in the DD nanocomposites should include the following characteristics: one is to create interfaces as much as possible and another is to decrease the permittivity gradient across interfaces. These characteristics can be realized by modifying the surface of inorganic nanoparticle and polymers on the basis of elaborate design and careful preparation. The final goal corresponding to modification is to ensure a homogenous dispersion of inorganic nanoparticles realizing more and uniform interfaces (what we want say here is: more interface is much better, interface with uniform thickness is much better) and to reduce the permittivity gradient across interfaces improving dielectric-dielectric coupling in the DD nanocomposites.

For the $\mathrm{CD}$ nanocomposites, the permittivity can be improved significantly at low loading of conductive fillers, which can be attributed to a dielectric scaling due to the percolation effect. ${ }^{31-35}$ The remarkable shortcomings are that the CD nanocomposites display a great dielectric loss, a low electrical breakdown field and the permittivity is extremely sensitive to the change in the concentration of conducing fillers near percolation threshold. These shortcomings limit the applications of $\mathrm{CD}$ nanocomposites in real engineering fields. In fact, like the permittivity gradient across interface in the DD nanocomposites, a great conductivity gradient across interface appears in this kind of CD nanocomposites as shown in Fig. 3(b). Unlike the dielectric gradient across interface, in place of the barrier action, it is just this large conductivity gradient resulting in a serious dielectric loss and a low electrical breakdown field. Perhaps, it is the main reason to be in charge of the shortcomings above. To overcome these shortcomings, similarly, the surface of conducting particle should be treated in order that the surface with low conductivity can be formed on the surface of nanoparticles with high conductivity. The final goal corresponding to surface treatment is to ensure a homogenous dispersion of conductive nanoparticles prohibiting a simple physical contact and to reduce the conductivity gradient across interfaces decreasing dielectric loss and improving electrical breakdown field.

To meet the viewpoints above, a more elaborate surface treatment of the nanoparticles is proposed here. A simple core-shell nanostructure (Fig. 4(a)) reported in past works

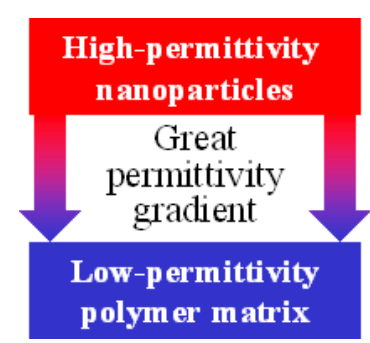

(a)

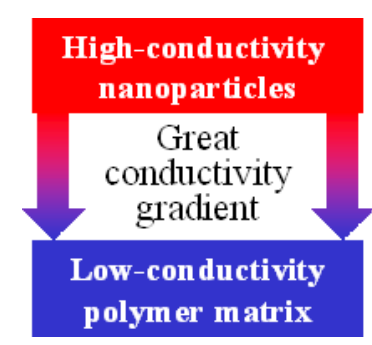

(b)
Fig. 3. (a) A great permittivity gradient in the DD nanocomposite and (b) a great conductivity gradient in the CD nanocomposite. may solve some questions shortcomings mentioned above in a certain extent. However, an entirely solving route is to design the nanoparticles with one-core-multi-shell nanostructure (Fig. 4(b)) and even the nanoparticles with an ideal structure (Fig. 4(c)) so that the permittivity/conductivity gradient across the interface between inorganic phase and polymer phase can be decreased. In addition, the characteristics of the outside shell should induce the homogeneous dispersion of modified nanoparticles and ensure an excellent interface interaction between nanoparticles and polymers.

\section{Some Reported Experimental Results}

\subsection{Surface treatment of dielectric nanoparticles in the DD nanocomposites}

For the DD nanocomposites, by means of $\mathrm{BaTiO}_{3}$ (BT), $\mathrm{PbTiO}_{3}$ (PT), $\mathrm{Pb}(\mathrm{Zr}, \mathrm{Ti}) \mathrm{O}_{3}(\mathrm{PZT}), \mathrm{Ba}_{x} \mathrm{Sr}_{1-x} \mathrm{TiO}_{3}$ (BST), $\mathrm{Pb}$ $\left(\mathrm{Mg}_{1 / 3} \mathrm{Nb}_{2 / 3}\right) \mathrm{O}_{3}$ (PMN) and $\mathrm{Pb}\left(\mathrm{Mg}_{1 / 3} \mathrm{Nb}_{2 / 3}\right) \mathrm{O}_{3}-\mathrm{PbTiO}_{3}$ (PMN-PT) with ferroelectric and relaxor ferroelectrics structure as functional fillers, ${ }^{36-52}$ all kinds of polymer composites with high permittivity have been studied in order to satisfy actual applications. In these $0-3$ composites, BT is widely used as fillers since it is relatively cheap and has been successfully prepared in different sizes from nanometer to micron. In the past works, the purpose of surface modification on BT nanoparticles is associated with the physical dispersion because the high surface energy of BT dielectric nanoparticles usually leads to agglomeration and phase separation from the polymer matrix, especially if they are forced into an incompatible matrix, yielding poor quality of films and weakened dielectric properties, such as high dielectric loss and low dielectric strength. ${ }^{23,53}$ It seems highly desirable to improve the compatibility between the filler and polymer matrix and consequently the energy storage performance of the nanocomposites (we also reported this for CCTO-polymer composite in our paper in Advanced Materials. ${ }^{17}$ Therefore, the action of interface between nanoparticles and polymers was mainly to improving the compatibility. For

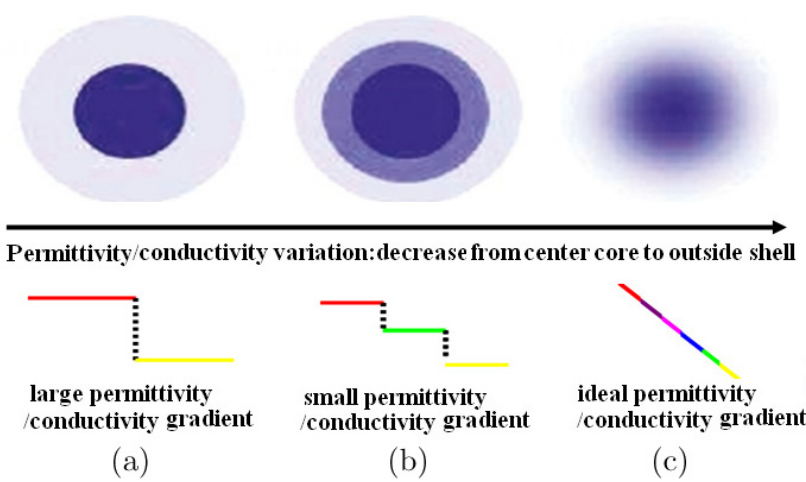

Fig. 4. (a) A simple core-shell nanostructure, (b) one-core-multishell nanostructure and (c) an ideal nanostructure of inorganic nanoparticles. 
example, Dang et al. reported an improved dielectric property in the BT/polymer nanocomposites when BT nanoparticles are chemically treated by employing a silane coupling agent (KH550) and $\mathrm{H}_{2} \mathrm{O}_{2}$, respectively. ${ }^{54,55}$ Ramesh et al. have reported BT/epoxy nanocomposite with pemittivity of 45 and an increased dielectric loss when BT nanoparticles are treated by trialkoxysilane surface modifiers. ${ }^{17} \mathrm{Kim}$ et al. have also demonstrated a strong surface binding of phosphonic acid ligands onto BT nanoparticles and examined the use of such specific surface functionalities to control BT/polymer interfacial interaction. ${ }^{53}$ In their work, the nanocomposites with 50 vol\% modified BT particles afforded a large permittivity $(\sim 37)$, reasonably low loss $(<0.007)$ and a high dielectric strength (over $200 \mathrm{MV} / \mathrm{m}$ ). Based on in situ process, Wang et al. successfully produced nanosized, ethylene diamine surface modified BT, so that it afford an enhancement in the dispersion of BT nanoparticles into organic solvents and subsequently led to a uniform polymer nanocomposite films. ${ }^{56}$

In the above reported researches, it can be seen that the characteristics of surface modifiers used to modify the surface of BT nanoparticles are in weak polarization and high electrical insulation. Though the uniform dispersion of nanoparticles and excellent compatibility between nanoparticles and polymers have been realized, the permittivity gradient across the interfaces is still extremely high so that the dielectric permittivity in the BT nanoparticle/polymer nanocomposites is still relatively low, even if the loading of the high-permittivity BT particles is high. Therefore, it is important that a suitable permittivity gradient across the interfaces should be designed by preparing the one-core-multi-shell inorganic nanoparticles as shown in Fig. 4(b). Perhaps, this is a crucial strategy to acquire the highpermittivity polymer composites.

\subsection{Surface treatment of conductive nanoparticles in the CD nanocomposites}

For the CD nanocomposites, the improvement of permittivity is mainly based on percolation effect, in which the concentration of the conductive particles is close to critical value and structurally the conductive networks consisting of the conductive particles are formed. Because of this reason, the fatal shortcomings of the conductive particle/polymer composites are an inevitably high dielectric loss and low electrical breakdown field due to the high leak of current if the unmodified conductive particles are dispersed into polymers directly. Another disadvantage is that the adjustment window of concentration variation on dielectric permittivity is very narrow due to a highly sensitive permittivity to the concentration variation of conductive particles around the percolation threshold.

In presently reported works, a few strategies have been proposed to prevent the direct contact between conductive particles near percolation threshold, with the aim of suppressing the high dielectric loss, maximizing the dielectric strength and also expanding the compositional windows. One straightforward approach is to introduce inter-particle barrier layer into percolative nanocomposites. As such, the tunneling current between neighboring conductive nanoparticles can be suppressed and hence leads to far lower dielectric loss and higher breakdown strength. The introduction of interfacial layer into percolative composites was first demonstrated by Wong and colleagues in metal/polymer composite, in which the surface of $100 \mathrm{~nm} \mathrm{Al}$ fillers was first self-passivated by a much thin layer $(2.8 \mathrm{~nm})$ of $\mathrm{Al}_{2} \mathrm{O}_{3}$, then compounded with epoxy to form percolative composites. ${ }^{57,58}$ As compared to the self-passivated inorganic shell, coating organic interfacial layers on the conductive nanoparticles has been demonstrated to be more versatile and allows for larger tunability in dielectric properties of percolative composites. Qi et al. have modulated $40 \mathrm{~nm} \mathrm{Ag}$ nanoparticles by a thin layer of mercaptosuccinic acid (MSA) molecules and then mixed them with epoxy by in situ polymerization process. ${ }^{59}$ The dielectric permittivity of the composites could increase up to 300 while the loss tangent still remained less than 0.05 . The presence of the organic coating makes the formation of conductive filler network very difficult, which thus significantly lowers the dielectric loss and affords a desirable high concentration tolerance for high permittivity. Therefore, a denser organic layer with sound structural integrity is much desirable to improve both low field and high field dielectric properties, thus, in turn, increase the energy density of percolative composites. In addition, Shen and Nan have also made such an effort in the $\mathrm{Ag}-\mathrm{C}$ core-shell nanoparticles/epoxy percolative composites with high permittivity $(\sim 450)$ and very low loss tangent $(\tan \delta<0.05) .{ }^{60,61}$ Fredin et al. enhanced the energy storage and suppressed the dielectric loss in an oxide core-shell structure nanocomposites by moderating internal surface area and increasing the shell thickness, and got substantial recoverable energy storage improvement in a percolative Al-polypropylene nanocomposites. ${ }^{62-64}$ The thickness of shells can be fine tuned to allow the possibility of tailoring the dielectric properties according to the specific requirements of practical applications.

Though the wonderful works mentioned above are very important to adjust the dielectric properties of the CD nanocomposites, not much attention was given to designing the conductivity gradient across the interfaces. It is believed that the CD nanocomposites can exhibit a much better dielectric performance if the interface is selected properly. One of the possible solutions is to design the one-core-multi-shell conductive particles with decreased conductivity from core to shell gradually as shown in Fig. 4(b) so that the low conductivity gradient across the inorganic particle/polymer interfaces can be reduced.

\section{Conclusion}

The interface characteristics between inorganic particle/ polymer nanocomposites with high permittivity are analyzed carefully in order to improve the dielectric properties further. 
The main motivation for the surface modification of nanoparticles in past works is to improve the uniform dispersion and prevent agglomeration of nanoparticles in order to realize a high permittivity of the particle/polymer composites. To certain extent, the interfacial interaction between particle phase and polymer phase is improved, which is valuable to the improvement of dielectric properties. To solve the present issues, it is proposed that the gradient variation in permittivity/ conductivity across the particle/polymer interfaces should be closely looked in the future. It is believed this is a crucial strategy to realize the high-performance high-permittivity polymer composites.

\section{Acknowledgments}

This work was financially supported by NSF of China (Grant No. 51377010), NSF of Beijing City (Grant No. 2122040), the National Basic Research Program of China (973 Program) (Grant No. 2014CB239503), and State Key Laboratory of Power System (SKLD11KZ04) in Tsinghua University.

\section{References}

${ }^{1}$ M. S. Whittingham, Materials challenges facing electrical energy storage, MRS Bull. 33, 411 (2008).

${ }^{2}$ J. S. Moya, S. Lopez-Esteban and C. Pecharroman, The challenge of ceramic/metal microcomposites and nanocomposites, Prog. Mater. Sci. 52, 1017 (2007).

${ }^{3} \mathrm{Q}$. Wang and L. Zhu, Polymer nanocomposites for electrical energy storage, J. Polym. Sci, Part B: Polym. Phys. 49, 1421 (2011).

${ }^{4}$ Y. Wang, X. Zhou, Q. Chen, B. Chu and Q. Zhang, The effect of barriers on electrical tree propagation in composite insulation materials, IEEE Trans. Dielectr. Electr. Insul. 17, 1036 (2010).

${ }^{5}$ Q. M. Zhang, H. F. Li, M. Poh, F. Xia, Z. Y. Cheng, H. S. Xu and C. Huang, An all-organic composite actuator material with a high dielectric constant, Nature 419, 284 (2002).

${ }^{6}$ Z. M. Dang, J. K. Yuan, J. W. Zha, T. Zhou, S. T. Li and G. H. Hu, Fundamentals, processes and applications of high-permittivity polymer-matrix composites, Prog. Mater. Sci. 57, 660 (2012).

${ }^{7}$ Z. M. Dang, T. Zhou, S. H. Yao, J. K. Yuan, J. W. Zha, H. T. Song, J. Y. Li, Q. Chen, W. T. Yang and J. Bai, Advanced Calcium Copper Titanate/Polyimide Functional Hybrid Films with High Dielectric Permittivity, Adv. Mater. 21, 2077 (2009).

${ }^{8}$ P. Barber, S. Balasubramanian, Y. Anguchamy, S. Gong, A. Wibowo, H. Gao, H. J. Ploehn and H. C. zur Loye, Polymer Composite and Nanocomposite Dielectric Materials for Pulse Power Energy Storage, Materials 2, 1697 (2009).

${ }^{9}$ E. Tuncer, I. Sauers, D. Randy James, A. R. Ellis, M. Parans Paranthaman, A. Goyal and K. L. More, Enhancement of dielectric strength in nanocomposites, Nanotechnology $\mathbf{1 8}$, 325704 (2007).

${ }^{10}$ M. Rahimabady, S. Chen, K. Yao, F. E. H. Tay and L. Lu, Thermal stability and electrical characteristics of ultrathin hafnium oxide gate dielectric reoxidized with rapid thermal annealing, Appl. Phys. Lett. 99, 142901 (2011).
${ }^{11}$ B. H. Fan, J. W. Zha, D. R. Wang, J. Zhao, Z. F. Zhang and Z. M. Dang, Preparation and dielectric behaviors of thermoplastic and thermosetting polymer nanocomposite films containing $\mathrm{BaTiO}_{3}$ nanoparticles with different diameters, Compus. Sci. Technol. 80, 66 (2013).

${ }^{12}$ Z. M. Dang, Y. Q. Lin, H. P. Xu, C. Y. Shi, S. T. Li and J. Bai, Fabrication and dielectric characterization of advanced $\mathrm{BaTiO}_{3} /$ polyimide nanocomposite films with high thermal stability, $A d v$. Funct. Mater. 18, 1509 (2008).

${ }^{13}$ J. K. Nelson and Y. Hu, Nanocomposite dielectrics - properties and implications, J. Phys. D, Appl. Phys. 38, 213 (2005).

${ }^{14}$ Y. Y. Sun, Z. Q. Zhang and C. P. Wong, Influence of interphase and moisture on the dielectric spectroscopy of epoxy/silica composites, Polymer 46, 2297 (2005).

${ }^{15}$ R. W. Coppard, J. Bowman, L. A. Dissado, S. M. Rowland and R. T. Rakowski, The effect of aluminium inclusions on the dielectric breakdown of polyethylene, J. Phys. D, Appl. Phys. 23, 1554 (1990).

${ }^{16}$ Z. M. Dang, H. P. Xu and H. Y. Wang, Significantly enhanced low-frequency dielectric permittivity in the BaTiO3/poly(vinylidene fluoride) nanocomposite, Appl. Phys. Lett. 90, 012901 (2007).

${ }^{17}$ S. Ramesh, B. A. Shutzberg, C. Huang, J. Gao and E. P. Giannelis, Dielectric nanocomposites for integral thin film capacitors: materials design, fabrication and integration issues, IEEE Trans. Adv. Packag. 26, 17 (2003).

${ }^{18}$ J. Zhang, E. Manias and C. A. Wilkie, Polymerically modified layered silicates: an effective route to nanocomposites, J. Nanosci. Nanotechnol. 8, 1597 (2008).

${ }^{19}$ E. Manias, Nanocomposites: Stiffer by design, Nature Mater. 6, 9 (2007).

${ }^{20}$ T. Tanaka, G. C. Montanari and R. Mulhaupt, Polymer nanocomposites as dielectrics and electrical insulation-perspectives for processing technologies, material characterization and future applications, IEEE Trans. Dielectr. Electr. Insul. 11, 763 (2004).

${ }^{21}$ J. Li, S. I. Seok, B. Chu, F. Dogan, Q. Zhang and Q. Wang, Nanocomposites of ferroelectric polymers with $\mathrm{TiO}_{2}$ nanoparticles exhibiting significantly enhanced electrical energy density, $A d v$. Mater. 21, 217 (2009).

${ }^{22}$ N. Guo, S. A. DiBenedetto, D. K. Kwon, L. Wang, M. T. Russell, M. T. Lanagan, A. Facchetti and T. J. Marks, Supported metallocene catalysis for in situ synthesis of high energy density metal oxide nanocomposites, J. Am. Chem. Soc. 129, 766 (2007).

${ }^{23}$ P. Kim, N. M. Doss, J. P. Tillotson, P. J. Hotchkiss, M. J. Pan, S. R. Marder, J. Li, J. P. Calame and J. W. Perry, High energy density nanocomposites based on surface-modified $\mathrm{BaTiO}_{3}$ and a ferroelectric polymer, ACS Nano 3, 2581 (2009).

${ }^{24}$ P. Murugaraj, D. Mainwaring and N. Mora-Huertas, Dielectric enhancement in polymer-nanoparticle composites through interphase polarizability, J. Appl. Phys. 98, 054304 (2005).

${ }^{25} \mathrm{~T}$. J. Lewis, Interfaces are the dominant feature of dielectrics at the nanometric level, IEEE Trans. Dielectr. Electr. Insul. 11, 739 (2004).

${ }^{26} \mathrm{P}$. Thomas, K. T. Varughese, K. Dwarakanath and K. B. R. Varma, Dielectric properties of Poly(vinylidene fluoride) $/ \mathrm{CaCu}_{3} \mathrm{Ti}_{4} \mathrm{O}_{12}$ composites, Compos. Sci. Technol. 70, 539 (2010).

${ }^{27}$ T. J. Lewis, Nanometric dielectrics, IEEE Trans. Dielectr. Electr. Insul. 1, 812 (1994). 
${ }^{28}$ L. Zhang and Z. Y. Cheng, Development of polymer-based 0-3 composites with high dielectric constant, J. Adv. Dielect. 4, 389 (2011).

${ }^{29}$ T. Hanemann and D. V. Szabo, Polymer-nanoparticle composites: From synthesis to modern applications, Materials 3, 3468 (2010).

${ }^{30}$ T. J. Lewis, Interfaces: Nanometric dielectrics, J. Phys. D, Appl. Phys. 38, 202 (2005).

${ }^{31}$ T. Tanaka, M. Kozako, N. Fuse and Y. Ohki, Dielectric nanocomposites with insulating properties, IEEE Trans. Dielectr. Electr. Insul. 12, 669 (2005).

${ }^{32}$ C. W. Nan, Y. Shen and J. Ma, Physical properties of composites near percolation, Annu. Rev. Mater. Res. 40, 131 (2010).

${ }^{33}$ Z. M. Dang, Y. H. Lin and C. W. Nan, Novel ferroelectric polymer composites with high dielectric constants, Adv. Mater. 15, 1625 (2003).

${ }^{34}$ J. K. Yuan, Z. M. Dang, S. H. Yao, J. W. Zha, T. Zhou, S. T. Li and J. Bai, Fabrication and dielectric properties of advanced high permittivity polyaniline/poly(vinylidene fluoride) nanohybrid films with high energy storage density, J. Mater. Chem. 20, 2441 (2010).

${ }^{35}$ Z. M. Dang, J. P. Wu, H. P. Xu, S. H. Yao, M. J. Jiang and J. Bai, Dielectric properties of upright carbon fiber filled poly(vinylidene fluoride) composite with low percolation threshold and weak temperature dependence, Appl. Phys. Lett. 91, 072912 (2007).

${ }^{36}$ Y. Deng, Y. Zhang, Y. Xiang, G. Wang and H. Xu, $\mathrm{Bi}_{2} \mathrm{~S}_{3}-\mathrm{BaTiO}_{3} /$ PVDF three-phase composites with high dielectric permittivity, J. Mater. Chem. 19, 2058 (2009).

${ }^{37}$ X. Huang, L. Xie, P. Jiang, G. Wang and F. Liu, Electrical, thermophysical and micromechanical properties of ethylene-vinyl acetate elastomer composites with surface modified $\mathrm{BaTiO}_{3}$ nanoparticles, J. Phys. D, Appl. Phys. 42, 245407 (2009).

${ }^{38}$ H. M. Jung, J. H. Kang, S. Y. Yang, J. C. Won and Y. S. Kim, Barium titanate nanoparticles with diblock copolymer shielding layers for high-energy density nanocomposites, Chem. Mater. 22, 450 (2010).

${ }^{39}$ Z. M. Dang, H. Y. Wang and H. P. Xu, Influence of silane coupling agent on morphology and dielectric property in $\mathrm{BaTiO}_{3} /$ polyvinylidene fluoride composites, Appl. Phys. Lett. 89, 112902 (2006).

${ }^{40}$ Y. Song, Y. Shen, H. Liu, Y. Lin, M. Li and C. W. Nan, Enhanced dielectric and ferroelectric properties induced by dopaminemodified $\mathrm{BaTiO}_{3}$ nanofibers in flexible poly(vinylidene fluoridetrifluoroethylene) nanocomposites, J. Mater. Chem. 22, 8063 (2012).

${ }^{41}$ Y. Song, Y. Shen, H. Liu, Y. Lin, M. Li and C. W. Nan, Improving the dielectric constants and breakdown strength of polymer composites: effects of the shape of the $\mathrm{BaTiO}_{3}$ nanoinclusions, surface modification and polymer matrix, J. Mater. Chem. 22, 16491 (2012).

${ }^{42}$ E. Tuncer, I. Sauers, D. R. James, A. R. Ellis and R. C. Duckworth, Electrical properties of percolative polystyrene/carbon nanofiber composites, IEEE Trans. Dielectr. Electr. Insul. 15, 236 (2008).

${ }^{43}$ X. L. Dou, X. L. Liu, Y. Zhang, H. Feng, J. F. Chen and S. Du, Improved dielectric strength of barium titanate-polyvinylidene fluoride nanocomposite, Appl. Phys. Lett. 95, 132904 (2009).

${ }^{44}$ W. Yang, S. Yu, R. Sun and R. Du, In situ observations of crack arrest and bridging by nanoscale twins in copper thin films, Acta Mater. 59, 5593 (2011).
${ }^{45}$ B. S. Prakash and K. B. R. Varma, Dielectric behavior of CCTO/ epoxy and Al-CCTO/epoxy composites, Comp. Sci. Technol. 67, 2363 (2007).

${ }^{46}$ F. Amaral, C. P. L. Rubinger, F. Henry, L. C. Costa, M. A. Valente and A. Barros-Timmons, Dielectric properties of polystyreneCCTO composite, J. Non-Cryst. Solids 354, 5321 (2008).

${ }^{47}$ H. Tang, Y. Lin, C. Andrews and H. A. Sodano, Nanocomposites with increased energy density through high aspect ratio PZT nanowires, Nanotechnology, 22, 015702 (2011).

${ }^{48}$ H. X. Tang and H. A. Sodano, Ultra high energy density nanocomposite capacitors with fast discharge using $\mathrm{Ba}_{0.2} \mathrm{Sr}_{0.8} \mathrm{TiO}_{3}$ nanowires, Nano Lett. 13, 1373 (2013).

${ }^{49}$ H. X. Tang, Y. R. Lin and H. A. Sodano, Enhanced energy storage in nanocomposite capacitors through aligned PZT nanowires by uniaxial strain assembly, Adv. Energy Mater. 2, 469 (2012).

${ }^{50}$ J. Yao, C. Xiong, L. Dong, C. Chen, Y. Lei, L. Chen, R. Li, Q. Zhu and $\mathrm{X}$. Liu, Direct and low energy electrolytic co-reduction of mixed oxides to zirconium-based multi-phase hydrogen storage alloys in molten salts, J. Mater. Chem. 19, 2817 (2009).

${ }^{51}$ K. Yu, H. Wang, Y. C. Zhou, Y. Y. Bai and Y. J. Niu, Enhanced dielectric properties of $\mathrm{BaTiO}_{3} /$ poly(vinylidene fluoride) nanocomposites for energy storage applications, J. Appl. Phys. 113, 034105 (2013).

${ }^{52}$ L. Y. Xie, X. Y. Huang, Y. H. Huang, K. Yang and P. K. Jiang, Core-shell structured hyperbranched aromatic polyamide/ $/ \mathrm{BaTiO}_{3}$ hybrid filler for poly(vinylidene fluoride-trifluoroethylene-chlorofluoroethylene) nanocomposites with the dielectric constant comparable to that of percolative composites, ACS Appl. Mater. Interfaces, 5, 1747 (2013).

${ }^{53}$ P. Kim, S. C. Jones, P. J. Hotchkiss, J. N. Haddock, B. Kippelen, S. R. Marder and J. W. Perry, Phosphonic acid-modified barium titanate polymer nanocomposites with high permittivity and dielectric strength, Adv. Mater. 19, 1001 (2007).

${ }^{54}$ Z. M. Dang, H. Y. Wang and H. P. Xu, Influence of silane coupling agent on morphology and dielectric property in $\mathrm{BaTiO}_{3} /$ polyvinylidene fluoride composites, Appl. Phys. Lett. 89, 112902 (2006).

${ }^{55}$ T. Zhou, J. W. Zha, R. Y. Cui, B. H. Fan and Z. M. Dang, Improving dielectric properties of $\mathrm{BaTiO}_{3} /$ ferroelectric polymer composites by employing surface hydroxylated $\mathrm{BaTiO}_{3}$ nanoparticles, ACS Appl. Mater. Inter. 3, 2184 (2011).

${ }^{56}$ J. Li, J. Claude, L. E. Norena-Franco, S. Seok and Q. Wang, Electrical energy storage in ferroelectric polymer nanocomposites containing surface-functionalized $\mathrm{BaTiO}_{3}$ nanoparticles, Chem. Mater. 20, 6304 (2008).

${ }^{57}$ J. W. Xu and C. P. Wong, Low-loss percolative dielectric composite, Appl. Phys. Lett. 87, 082907 (2005).

${ }^{58}$ J. W. Xu, K. S. Moon, C. Tison and C. P. Wong, A novel aluminum-filled composite dielectric for embedded passive applications, IEEE Trans. Adv. Packag. 29, 295 (2006).

${ }^{59}$ L. Qi, B. I. Lee, S. H. Chen, W. D. Samuels and G. J. Exarhos, High-dielectric-constant silver-epoxy composites as embedded dielectrics, Adv. Mater. 17, 1777 (2005).

${ }^{60}$ Y. Shen, Y. H. Lin, M. Li and C. W. Nan, High dielectric performance of polymer composite films induced by a percolating interparticle barrier layer, Adv. Mater. 19, 1418 (2007).

${ }^{61}$ Y. Shen, Y. Lin and C. W. Nan, Interfacial effect on dielectric properties of polymer nanocomposites filled with core/shellstructured particles, Adv. Funct. Mater. 17, 2405 (2007). 
${ }^{62}$ L. A. Fredin, Z. Li, M. A. Ratner, M. T. Lanagan and T. J. Marks, Enhanced energy storage and suppressed dielectric loss in oxide core-shell-polyolefin nanocomposites by moderating internal surface area and increasing shell thickness, Adv. Mater. 24, 5946 (2012).

${ }^{63}$ L. A. Fredin, Z. Li, M. T. Lanagan, M. A. Ratner and T. J. Marks, Substantial recoverable energy storage in percolative metallic aluminum-polypropylene nanocomposites, Adv. Funct. Mater. 23 , 3560 (2013).

${ }^{64}$ L. A. Fredin, Z. Li, M. T. Lanagan, M. A. Ratner and T. J. Marks, Sustainable high capacitance at high frequencies: Metallic aluminum-polypropylene nanocomposites, ACS Nano 7, 396 (2013). 